\title{
Réflexion sur les perceptions, conceptions, représentations et affections, ou la quadrature des approches qualitatives en géographie
}

Reflection on perceptions, conceptions, representations and affections, or the quadrature of qualitative methods in geography

\section{Una reflexión sobre las percepciones, concepciones, representaciones y afectos, o la cuadratura de los enfoques cualitativos en Geografía}

\section{Mario BÉDARD}

Volume 60, numéro 171, décembre 2016

Version originale soumise en juillet 2016. Version révisée reçue en janvier 2017.

URI : https://id.erudit.org/iderudit/1041221ar

DOI : https://doi.org/10.7202/1041221ar

Aller au sommaire du numéro

Éditeur(s)

Département de géographie de l’Université Laval

ISSN

0007-9766 (imprimé)

1708-8968 (numérique)

Découvrir la revue

Citer cet article

BÉDARD, M. (2016). Réflexion sur les perceptions, conceptions, représentations et affections, ou la quadrature des approches qualitatives en géographie.

Cahiers de géographie du Québec, 60(171), 531-549.

https://doi.org/10.7202/1041221ar

\section{Résumé de l'article}

Les chercheurs en géographie humaine tentent de saisir le plus finement possible la nature des relations que nous entretenons avec la Nature et le Territoire à partir de ce qu'en " pensent » les divers acteurs ou groupes d'acteurs concernés. Or, ces " pensées " émanent de registres de signification fort distincts selon que ceux-ci résultent de la perception, de la représentation, de la conception ou de l'affection. Soit quatre processus souvent confondus, par suite du manque d'amplitude et d'acuité du regard exercé et, surtout, parce qu'ils ne sont pas abordés et examinés communément. Cela n'est pas sans conséquences sur la cohérence de toute démarche référant à l'un ou l'autre de ces modes relationnels pour saisir les « opinions » des acteurs, puis sur la validité des résultats obtenus. Et c'est pour tenter de résoudre quelque peu ces difficultés dans notre compréhension des relations subjectives que l'être humain entretient avec son environnement naturel ou bâti que nous cherchons, dans ce texte, à dégager et articuler les principales modalités et finalités à l'oeuvre au sein de la perception, de la représentation, de la conception et de l'affection. 


\title{
Réflexion sur les perceptions, conceptions, représentations et affections, ou la quadrature des approches qualitatives en géographie ${ }^{1}$
}

\author{
Reflection on perceptions, conceptions, \\ representations and affections, or the \\ quadrature of qualitative methods in geography \\ Una reflexión sobre las percepciones, concepciones, \\ representaciones y afectos, o la cuadratura de los \\ enfoques cualitativos en Geografía
}

\author{
Mario BÉDARD \\ Département de géographie \\ Université du Québec à Montréal \\ Centre de recherches sur les innovations sociales \\ (CRISES) \\ Bedard.Mario@uqam.ca
}

\section{Résumé}

Les chercheurs en géographie humaine tentent de saisir le plus finement possible la nature des relations que nous entretenons avec la Nature et le Territoire à partir de ce qu'en "pensent» les divers acteurs ou groupes d'acteurs concernés. Or, ces "pensées» émanent de registres de signification fort distincts selon que ceux-ci résultent de la perception, de la représentation, de la conception ou de l'affection. Soit quatre processus souvent confondus, par suite du manque d'amplitude et d'acuité du regard exercé et, surtout, parce qu'ils ne sont pas abordés et examinés communément. Cela n'est pas sans conséquences sur la cohérence de toute démarche référant à l'un ou l'autre de ces modes relationnels pour saisir les "opinions» des acteurs, puis sur la validité des résultats obtenus. Et c'est pour tenter de résoudre quelque peu ces difficultés dans notre compréhension des relations subjectives que l'être humain entretient avec son environnement naturel ou bâti que nous cherchons, dans ce texte, à dégager et articuler les principales modalités et finalités à l'œuvre au sein de la perception, de la représentation, de la conception et de l'affection.

\section{Mots-clés}

Perception, représentation, conception, affection, modes relationnels, registres de signifiance.

\begin{abstract}
Human geographers devote themselves to understanding as subtly as possible the nature of the relationships that we have with Nature and Territory, by exploring what the concerned stakeholders or groups of stakeholders "think" of those relationships. And yet, these "thoughts" can come from very distinct registers of significance, depending on whether they are the result of perceptions, representations, conceptions or affections. These processes are often used interchangeably, which creates a problem attributable to the limited range and acuteness of geographers' analysis and, more importantly, to the lack of an approach which adequately integrates all of them. This issue is significant since it diminishes the coherence of reflections which refer to one of these four processes, especially when it comes to truly grasping the "opinions" of the stakeholders involved and assessing the validity of the results. In order to resolve these difficulties and shortcomings in our comprehension of the subjective relationships that human beings have with their natural or constructed environments, this article will try to highlight and articulate the principal modalities and aims structuring perceptions, representations, conceptions and affections.
\end{abstract}

\section{Keywords}

Perception, representation, conception, affection, relational modes, significance registers.

1 Le terme «quadrature» est employé ici au sens de faire le tour de la question. 


\section{Resumen}

Los investigadores en Geografía Humana tratan de captar con la mayor sutileza, la clase de nuestras relaciones con la Naturaleza y con el Territorio, comenzado por lo que "piensan" los actores o grupos de actores involucrados. Ahora bien, esos "pensamientos" surgen de muy distintos registros de significación, según que emanen de la percepción, de la representación, de la concepción o del afecto. Eh ahí cuatro procesos que se confunden muy a menudo, debido a la estrechez y a la torpeza de la observación, y, sobre todo, porque de ordinario no se los enfoca ni se los examina. Ello tiene consecuencias sobre la coherencia de todo proceso concerniente a uno u otro de tales modos relacionales para captar las "opiniones" de los actores, luego, sobre la validez de los resultados obtenidos. Con el fin de tratar de resolver, al menos en parte, esas dificultades, en la comprensión de las relaciones subjetivas que el ser humano tiene con su medio natural o construido, buscamos, en este texto, despejar y articular las modalidades y finalidades principales dentro de la percepción, de la representación, de la concepción y del afecto.

\section{Palabras claves}

Percepción, representación, concepción, afecto, modos relacionales, registros de significado.

\section{Introduction}

La géographie humaine, quelle que soit sa sous-discipline (géographie culturelle, économique, politique, rurale, sociale, urbaine ou autres), tout comme l'ensemble des sciences sociales ou la philosophie, interroge toujours d'une façon ou d'une autre les multiples tenants (dont les idéologies et valeurs qui les animent et structurent) ou aboutissants (dont les schémas d'aménagement, plans d'urbanisme, politiques, lois ou règlements qui les façonnent) des rapports Homme/Nature, Espace/Société et Territoire/Culture. ${ }^{2}$ Tous les chercheurs en géographie humaine s'évertuent en effet d'une manière plus ou moins explicite à saisir la nature des relations qu'un individu, un groupe, une communauté, une collectivité ou une société entretient vis-à-vis la Nature, le Territoire, et ce, tout spécialement dans le cadre de l'évolution de la (ou des) fonction(s) qu'il ou elle prête à un lieu, un territoire ou un espace, et qu'interpelle tout particulièrement un projet (grand projet urbain, usine d'épuration des eaux usées, loi modifiant les règles de zonage en milieu rural...) ou une métamorphose lente ou subite, souhaitée ou accidentelle (nouvelle autoroute, verdissement des toitures en milieu urbain, modification du rôle d'évaluation foncière, reconnaissance des droits autochtones, inondation, glissement de terrain, etc.). Pour ce faire, l'une des approches privilégiées, notamment depuis l'avènement des courants de pensée critiques et postmodernes, est d'y réfléchir «directement» à partir de ce qu'en «pensent» les divers acteurs ou groupes d'acteurs concernés, qu'il s'agisse d'opinions ou d'avis émis au Je individuel, au Nous clanique ou communautaire, sinon au Nous collectif et sociétal.

2 Si cette préoccupation est aussi de plus en plus sensible en géographie physique et dans le champ des systèmes d'information géographique (SIG) dès lors qu'on s'intéresse aux causes et conséquences de leurs interventions ou lectures de leurs objets d'étude, il reste que c'est surtout en géographie humaine que cette interrogation sur les tenants et aboutissants prévaut, tant et si bien que c'est en ce domaine que la géographie cherche tout particulièrement à se donner les moyens les plus performants à cet égard. 
Or, ces "pensées» émanent de registres de signification fort distincts selon que ceux-ci résultent de perceptions, de représentations, de conceptions ou d'affections. ${ }^{3}$ Ces processus en appellent en effet à différents types de rapports au Réel qui, pour proches et liés qu'ils soient (tout n'est-il pas construction de l'esprit?), demeurent foncièrement autres en vertu de modalités et de finalités propres à chacun. Et il en est ainsi, chercherons-nous à démontrer, dans la mesure où ces quatre modes relationnels reposent sur une charge de sens variable (i) compte tenu de leurs objets spécifiques et de leurs fonctions d'évocation, puis (ii) s'ils procèdent d'une appréciation intérieure ou extérieure du phénomène en cause, un positionnement changeant selon le degré d'implication et de compréhension des acteurs concernés ou visés, mais encore selon le type de référentiel et d’imagination à l'œuvre.

Cela précisé, pour arriver à plus exactement cibler les acteurs souhaités et leur mode d’intellection du phénomène étudié, soit deux décisions qui ne sont pas sans impact sur le type d'échantillonnage et la grosseur de l'échantillon de toute démarche de recherche, de même que sur les modes de collecte, de traitement et d'analyse des données, il faut selon nous pouvoir sciemment choisir si on doit examiner leurs perceptions, représentations, conceptions ou affections, sinon diverses combinaisons de ces éléments. Or, ces nuances sont rares, la très grande majorité des dictionnaires, encyclopédies et travaux de géographes (ou autres) étudiant les pensées des acteurs en cause, sinon ce qu'ils disent nommément être leurs perceptions, représentations, conceptions ou affections, n’ayant pas ces préoccupations.

\section{Bilan des entendements et usages des processus de perception, représentation, conception et affection}

Sensible au fait qu'en se concentrant, tels ses courants de pensée scientistes antérieurs, sur les seuls aspects matériels ou objectifs de notre condition géographique, elle ne peut qu'en promulguer une compréhension et une gestion partielles et limitatives (Bailly, 1974), la géographie s’intéresse, plus spécifiquement depuis le début des années 1970, aux relations subjectives que l'être humain entretient avec son environnement naturel ou bâti. Pour ce faire, elle a développé trois grandes approches.

3 Tout comme Spinoza (1677/1993), nous emploierons le terme affection pour désigner cet autre processus d'intellection relationnelle par lequel l'indicible peut être saisi pour étudier les «forces vives» qui influent sur notre expérience d'un phénomène (Thrift, 2008) (atmosphère, ambiance, etc.) et, dès lors, sur le sens qu'on lui confère puis sur les pensées et actions qui s'ensuivent. Si nous sommes conscient que ce terme peut prêter à confusion, compte tenu des autres significations plus usuelles qui lui sont associées (sentiment ou pathologie), il demeure à notre avis plus approprié pour évoquer le processus ici visé que les termes affect ou ressenti, sinon sensation ou émotion, certes davantage répandus, n'expriment que partiellement, voire partialement. En effet, si ces derniers y participent également, ils sont et demeurent toutefois plus spécifiques, c'est-à-dire associés à l'une ou l'autre modalité ou finalité du processus qui ne leur est pas ainsi réductible (McCormack, 2003). Attendu que nous y reviendrons plus amplement, soulignons pour l'instant que nous n'avons pas retenu ces termes comme appellation de ce mode relationnel car l'affect n'en est qu'un incitatif, le ressenti une résultante, puis parce que l'affection recouvre les sensation et émotion, que ce soit à titre d'incitatifs comme acteurs-actants et/ou de résultantes. 
Du nombre, signalons d'abord le travail de la géographie humaniste ${ }^{4}$ qui, à la suite des travaux de Lynch (1960) et de Moles et Rohmer (1972) en psychologie de l'environnement, mais encore des géographes précurseurs que furent Lowenthal (1961), Kirk (1963), Gould (1966) et Appleyard (1970), investigue tout spécialement les perceptions que nous avons de notre condition territoriale, cela notamment à partir d'une lecture cherchant à (r)établir l'importance du sujet, et donc du sens que celui-ci confère aux phénomènes avec lesquels il est en relation, par exemple aux lieux et au sentiment d'appartenance qu'il peut développer à leur endroit (Tuan, 1971, 1974, 1977 ; Buttimer, 1974, 1976; Seamon, 1979; Ley, 1980; Bailly, 1981). ${ }^{5}$

Notons ensuite la contribution de la géographie poststructuraliste, à l'œuvre depuis les années 1990 et qui, cherchant à mieux composer avec un univers plus complexe et dynamique qu'initialement anticipé, explore plus spécialement les représentations qu'elle dit plus significatives compte tenu de leur plus grand apport référentiel, en déconstruisant le continuum texte-discours-métaphore pour mieux illustrer en quoi notre monde et le sens qu'on lui prête sont d'abord des constructions de notre crû, soulignant pour ce faire toute l'importance de l'imaginaire et des symboles (Philo, 1992 ; Dixon et Jones, 1998; Doel, 1999).

Il y a enfin l'entreprise poursuivie par la géographie non représentationnelle du renouveau culturel (cultural turn). Faisant sienne l'idée de Derrida (1978) et de Baudrillard (1981) selon laquelle la représentation n'est pas tant une chose qu'un acte produisant la réalité pour mieux considérer l'intersubjectivité de notre condition relationnelle (Bondi, 2005) et ainsi préconiser une pensée géographique plus complète (Davidson et Smith, 2009), voire plus empathique (Creswell, 2012), cette géographie non représentationnelle du renouveau culturel réfléchit sur l'incidence des affections qui, au-delà et en-deçà des perceptions et des représentations, animent et signifient tout autant sinon plus, soutient-elle, nos modes d'habiter et nos milieux de vie, car elles aussi porteuses de sens (Thrift, 2004; Anderson, 2006).

Or, pour fécond et heureux qu'il soit ou semble être dans les champs de chacune de ces approches, cet affinement demeure inachevé et perfectible. Ce problème est attribuable, à prime abord, (i) au manque d'amplitude et d'acuité du regard exercé, rares étant les travaux

1. qui ne souffrent pas d'imprécisions ou de méconnaissances alors qu'ils usent de définitions ou de paramètres flous et inappropriés qui confondent leurs entendements et usages ou les agrègent sous l'une ou l'autre dénomination, tels des synonymes, et prêtent par exemple à l'affection ou à la perception des éléments propres à la représentation (Bueno, 2013 ; Marková, 2015), cherchant donc l'une mais traitant de l'autre (Bailly, 1981);

2. qui considèrent plus d'un de ces processus relationnels, si ce n'est pour justifier la valeur ou plutôt la suprématie de celui que tel ou tel courant de pensée préconise (Drestke, 2000; Smith 2002);

3. voire qui approfondissent les conceptions, absentes de la précédente nomenclature, pouvant aussi être faites de nos relations subjectives à la Nature et au Territoire.

4 Les trois grandes approches ici évoquées approfondissant nos relations subjectives à la Nature, au Territoire et à la Société. Elles sont, pour les besoins de notre propos, largement contextuelles, résumées à une peau de chagrin qui, en aucune façon, ne peut témoigner de la richesse de leurs apports respectifs.

5 Ne sont mentionnés que quelques-uns des géographes qui ont le plus contribué à la réflexion sur ces processus, attendu que les trois approches présentées ici de manière séquentielle prévalent toujours. 
Ce problème d'amplitude et d'acuité est encore et surtout imputable (ii) au fait que ces quatre processus ne sont pas abordés et examinés communément, puis systématisés et articulés ensemble comme autant de modes relationnels et de registres de signifiance complémentaires. Il en est ainsi, à notre avis, car cet affinement nous apparaît être plus fortuit et conjoncturel que volontaire et structurel, c'est-à-dire plus associé à des repositionnements théoriques spécifiques, si ce n'est à des renouveaux paradigmatiques (ou dits tels) associés au passage d'un courant de pensée à un autre, tels des mouvements de balancier où l'on passe d'un schéma explicatif à un autre, jugé plus performant, sinon plus vrai, et ce, sans vraiment envisager et traiter de manière globale et concertée les autres processus. Cela n'est pas sans conséquences sur la cohérence de toute démarche référant à l'un ou l'autre de ces processus pour saisir les opinions des acteurs, puis sur la validité des résultats obtenus, notamment en termes de précision, et donc de représentativité.

Ces limites sont toutes aussi sensibles chez les dictionnaires et encyclopédies géographiques auxquels réfèrent en premier lieu les géographes lorsqu’ils s’intéressent à pareils processus. Nous en voulons pour preuve la quinzaine d'ouvrages consultés dont les entrées reposent sur certaines des idées et postures ci-dessus dégagées (écrites qu'elles sont souvent par les mêmes auteurs ou se rapportant à leurs textes), tant et si bien qu'ils ne font que perpétuer les mêmes problèmes d'amplitude et d'acuité du regard, mais surtout d'absence de lecture, systématisation et articulation communes auxquelles se prêtent pourtant fort bien ces types d'écrit. Alors qu'ils devraient être les abécédaires et grammaires de la réflexion géographique, ces dictionnaires et encyclopédies se révèlent peu concernés par ces processus, sinon que par la représentation et, dans une moindre mesure, la perception. En effet, si seuls quelques-uns n'en abordent aucun (Wackerman, 2005; Baud et al., 2013), la plupart ne traitent explicitement que d'un seul (perception pour George et Verger, 2004; Mayer et Roche, 2012; représentation pour Bailly, Ferras et Pumain, 1995; Gregory et al., 2009; Castree et al., 2013) ou de la perception ET de la représentation (Douglas et al, 2002 ; Bailly, 2005 ; Brunet, Ferras et Théry, 2005; Kitchin et Thrift, 2009; Mayhew, 2009; Warf, 2010; Lévy et Lussault, 2013).

Généralement, la perception y est très succinctement définie et appelle des précisions supplémentaires («système relationnel impliquant, dans une même tension intentionnelle, le monde vécu, les sens et la conscience» (Di Méo, 2013 : 759)), voire malhabilement («the manner by which we make sense of the world» (Mayhew, 2009: 374)) ou de manière trop simplificatrice («Image de l'espace familier, conçue par ceux qui y vivent et le fréquentent» (George et Verger, 2004: 316), vague («présentation de quelque chose à l'esprit. En général, forme que prend dans l'intellect une idée, un phénomène, un objet, un espace» (Brunet, Ferras et Théry, 2005 : 428)) ou fautive («représentation que l'on se fait d'un espace géographique et de son contenu» (Mayer et Roche, 2012: 262)). La représentation, pour davantage détaillée qu'elle soit, demeure plus considérée comme un objet que comme un processus. Les trois exceptions sont Debarbieux (2013: 867) qui souligne que cette notion réfère aussi bien à un processus («élaboration de la configuration») qu'à un objet ("configuration stabilisée»), et s'avère donc tout aussi bien matérielle qu'immatérielle, Kobayashi (2009: 348-349) qui distingue les représentations qui évoquent et confortent («that speak of») des re-présentations qui connotent et mobilisent («that speak for») afin d’illustrer en quoi ce processus et son objet sont foncièrement complexes et évolutifs, puis Castree, Kitchin et Rogers pour qui, verbe et nom comme processus et produit, la représentation propose des «acts of substitution and translation in which different 
people make sense of the world» (2013: 431). Nul dictionnaire ou encyclopédie ne décrit comme tel le processus d'affection, si ce n'est parfois allusivement par l'affect, l'émotion ou le sensitif, c'est-à-dire en référence là encore à l'objet plutôt qu'au processus (Gregory et al., 2009; Mayhew, 2009; Castree, Kitchin et Rogers, 2013). Enfin, comme pour les travaux de recherche précédemment relatés, rien n’est précisé sur la conception, si ce n'est de manière très distante par le concept ou la conceptualisation, et donc dans un tout autre horizon de sens.

Somme toute, lorsque l'un ou l'autre de ces processus est déconstruit, cela demeure assez peu approfondi et rarement, si ce n'est jamais, en lien véritable avec les trois autres. Et si Kobayashi (2009) étudie davantage la relation entre représentation, affect et émotion, ces deux derniers sont abordés non pas comme des extensions de la représentation ou éléments d'un autre processus, mais plutôt comme parties prenantes d'une représentation oppositionnelle sur laquelle elle ne développe pas. C'est dire que ces dictionnaires et encyclopédies, alors même qu'ils devraient constituer des sommes des clés tant conceptuelles que processuelles de la géographie, souffrent des mêmes insuffisances (et plus) que la littérature spécialisée explorant nommément les perception, représentation, conception ou affection.

Or, ces difficultés tout spécialement préjudiciables pourraient être évitées si on savait mieux d'entrée de jeu ce dont il est question (soit le type de relations en cause) et donc quel(s) processus est (sont) à investiguer au su de la problématique posée. Et c'est pour tenter de résoudre quelque peu ces difficultés que nous avons rédigé le présent texte, présumant qu'une plus subtile intelligence des principales modalités et finalités à l'œuvre au sein des perception, représentation, conception et affection pourrait permettre de les employer de manière davantage appropriée et, de là, peutêtre, en arriver à une compréhension plus juste de la complexité et de la dynamique de la charge de signifiance immanente aux rapports Homme/Nature, Espace/Société et Territoire/Culture qu'explore la géographie.

Certes, la déconstruction qu'appelle pareil exercice d'«étoffement» épistémologique et d'articulation méthodologique implique une mise à distance et un grossissement des traits tout d'artifices avec ce que cela peut supposer d'arbitraire ou de bancal. Nous faisons toutefois le pari que cela sera de peu de conséquences face aux bénéfices pouvant en être retirés. Précisions d'autre part que, pour faciliter la compréhension des nuances que nous chercherons à souligner ici, nous référerons à un même exemple, soit les perceptions, représentations, conceptions ou affections qui peuvent être faites du sapin baumier, largement connu qu'est cet arbre, sinon l'idée qu'on s'en fait, par l'ensemble des populations. De la même façon, nous procéderons chaque fois selon une même démarche où, après une courte caractérisation du processus à l'étude, seront repris et détaillés ses principaux attributs. Soulignons également que si, pour cela, nous emprunterons à d'autres sciences, notre réflexion sera d'abord et avant tout géographique, c'est-à-dire faite par et pour les géographes. Notons enfin que les quatre processus scrutés ne créent ni le phénomène observé, ni le sujet mis en tension, ni la relation entre les deux, et ce, même lorsqu'articulés les uns aux autres. Ces phénomènes, sujets et relations sont et demeurent en effet fondamentalement distincts et pluriels, tant et si bien que les modes relationnels et registres de signifiance explorés illustreront, au mieux, cette pluralité et, dès lors, la profondeur comme la richesse de notre condition géographique et territoriale. Cela n'est toutefois pas rien, car cette plus grande conscience du registre des significations que nous accordons à nos relations à la Nature et au Territoire ne peut que bonifier le travail du géographe. 


\section{Les quatre processus relationnels des approches qualitatives}

\section{Perception}

La perception est un processus relationnel primaire qui procède (i) par l'action de nos sens, et donc en présence du phénomène, puis (ii) d'un décodage contigu et immédiat afférent à une signification basique en vertu d'un filtre cognitif dénotatif permettant de traduire et structurer les données sensorielles pour identifier et nommer ledit phénomène, c'est-à-dire pour le (re)connaître.

De manière plus détaillée, la perception est un mode de compréhension mi-objectif et mi-subjectif qui s’intéresse au sensitif, soit à ce qui peut être détecté et distingué de manière «certaine». Pour ce faire, la perception se réalise en deux temps concomitants de détection, puis d'identification (Alston, 2005). Tout d'abord, par l'intermédiaire du goût, de l'odorat, de l'ouïe, du toucher ou de la vue, elle cherche à (re)constituer mécaniquement tout phénomène matériel (naturel ou anthropique) devant lequel nous sommes, c'est-à-dire à établir sa réalité, son existence en vertu d'une saisie brute d’informations. Puis, de manière quasi simultanée à cette «réception intérieure, somme toute passive car automatique, spontanée et [...] objective des objets de notre monde» (Lalande, 1926/2002: 754), ce même processus s'emploie à (re)connaître ce phénomène. C'est-à-dire à l'identifier en vertu d'un principe réflexif de décodage (Morin, 2003) qui, par association au moyen d'archétypes (Bailly, 1974) et par contextualisation vis-à-vis son environnement (Bueno, 2013), le signifie (Rousseau, 1782 / 1995) et le nomme comme un phénomène (Marková, 2015) appartenant à une catégorie d'objets spécifiques ou inclus dans une idée générale. Nous pouvons ainsi voir un sapin baumier sans être conscients qu'il en est un (phase I). Pour y parvenir, il nous faut savoir, fût-ce de manière vague et intuitive (phase II), ce qu'est un sapin baumier à partir de l'odeur ou de la saveur de sa résine, de la forme de ses branches, cônes et aiguilles, de la rugosité de son écorce, etc. pour le différencier (figure 1).

Si ce second acte dénotatif grâce auquel sont caractérisées et organisées nos données sensibles illustre bien que la perception n'est pas qu'un automatisme sensoriel, il souligne également qu'elle est une fonction mineure d'entendement (Descartes, 1649/1978) subjectif (Kant, 1781/1998) car, collant à la concrétude des choses et à la véracité des idées établies à leur égard, elle procède sans réelle imagination ou distanciation comparativement à la représentation, la conception et l'affection à venir, si ce n'est au moyen d'un imaginaire reproducteur qui décline le champ circonscrit d'une compréhension générique suffisante pour discriminer les phénomènes (Pacherie, 2013). Cela dit, la géographie, en tant que science sociale, n'examine pas vraiment les divers mécanismes physiologiques à l'œuvre lors de la perception (phase I). Elle investigue plutôt le second temps cognitif de ce processus par lequel ce que nos sens dégagent est discerné et situé.

\section{Représentation}

La représentation est un processus relationnel secondaire d'évocation et d'interprétation qui procède par le seul travail de notre esprit, cela, en vertu d'une opération connotative permettant de qualifier le sens qu'on prête audit phénomène et à la relation antécédente qu'on entretient à son égard, c'est-à-dire pour le comprendre. 
Figure 1 Illustrations de quelques-uns des éléments perceptibles d'un sapin baumier

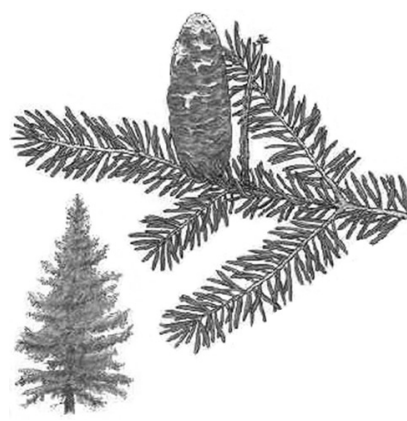

Source: Leboeuf, 2016: 112-113

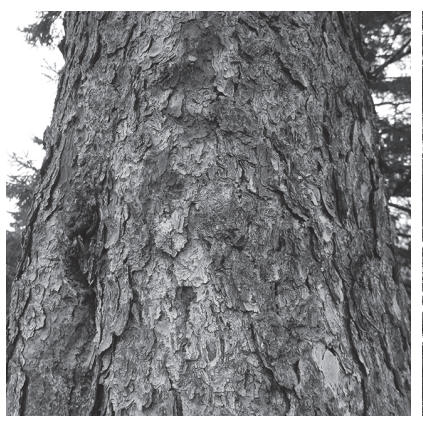

Source: Bédard, 2016

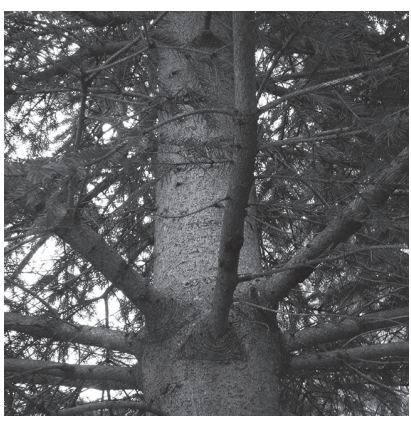

Source: Bédard, 2016

Autrement dit, s'effectuant en l'absence du phénomène, la représentation est un mode d'entendement indirect et abstrait grâce auquel l'être humain non seulement rend à nouveau sensible - une opération de redite que souligne le préfixe re - un phénomène oublié ou lointain qu'il a déjà perçu en vertu d'une image, d'un signe, d'un mot le rappelant, mais qui, encore et surtout, exprime par l'encodage que permettent cette image, ce signe et ce mot, ce que ce phénomène signifie, non plus en lui-même, mais par et pour ce même individu. Il en est ainsi parce que cette image, ce signe ou ce mot participent d'un imaginaire (re)créateur imaginalisant (Durand, 1968; Wunenburger, 1991), soit un imaginaire heuristique et transcendant en ceci qu'il interpelle par leur truchement un surcroît de sens autrement inaccessible grâce auquel l'être humain peut évoquer par connotation toute l'ampleur, l'importance et la singularité de la charge de signifiance qu'il attribue à ce phénomène. Tant inférentiel, façonné qu'il est par une mémoire endocentrée qui donne à voir, que référentiel, car aussi modulé par une mise en images ou en mots exocentrée (Bailly, 1985 ; Drestke, 1995), cet imaginaire use d'un double système connotatif (Kosslyn, 1980; Alston, 2005) explorant et valorisant la relation qui prévaut entre le représentant et le représenté, et donc plus particulièrement la signification tant mnémonique que sémiotique ou symbolique que le premier confère au second.

En donnant ainsi accès à une couche de sens plus étoffée et déliée, le processus de (re) construction et d'expression du sujet qu'opère la représentation s'avère fondamentalement qualificatif parce qu'il émane d'une interprétation spécifique qui participe d'une fonction d'appropriation et de signification œuvrant au plus près du Je car tributaire de son jugement de valeurs et, dès lors, présumé plus en phase avec les relations foncières qu'un individu entretient avec la Nature, le Territoire, la Société, l'Autre somme toute. ${ }^{6}$ Soit une intériorisation qui, au final et selon un nombre sans cesse croissant de géographes et autres scientifiques des sciences sociales, régit fondamentalement «notre relation au monde et aux autres» (Jodelet, 1994: 37). Toute représentation d'un sapin baumier repose en effet sur une recréation non pas de ce que ce sapin est, objectivement, mais bien de ce qu'il évoque pour un individu, une famille, une communauté, que ce soit à partir de traits marquants - ou présumés tels - (forme du port de ses branches) ou contextuels (typicité environnementale des endroits où on le retrouve(rait)), voire de ce

6 Ce Je, s'il est certes individuel car seul capable de libre arbitre et donc, au final, capable de décider de la signification à accorder à un phénomène, n'en est pas moins modulé en partie par divers acquis ou valeurs qui lui sont "extérieurs», soient-ils claniques, communautaires, collectifs ou sociétaux, et auxquels il adhère plus ou moins consciemment, une adhésion par ailleurs si forte que ces acquis ou valeurs «extérieurs» lui sont indissociables. 
qu'il incarne en fonction des usages qu'on en fait ou des significations qu'on lui prête. Il s'ensuit que toute représentation (figure 2) d'un sapin baumier nous permet d'identifier le type d'interprétation qui en est faite, puis de comprendre le sens et la valeur qu'on lui accorde (esthétique, éthique, utilitaire, symbolique, etc. - un beau sapin baumier, un gros sapin baumier, un bon ou authentique sapin baumier, etc.).

\section{Figure 2 Représentations d'un sapin baumier}

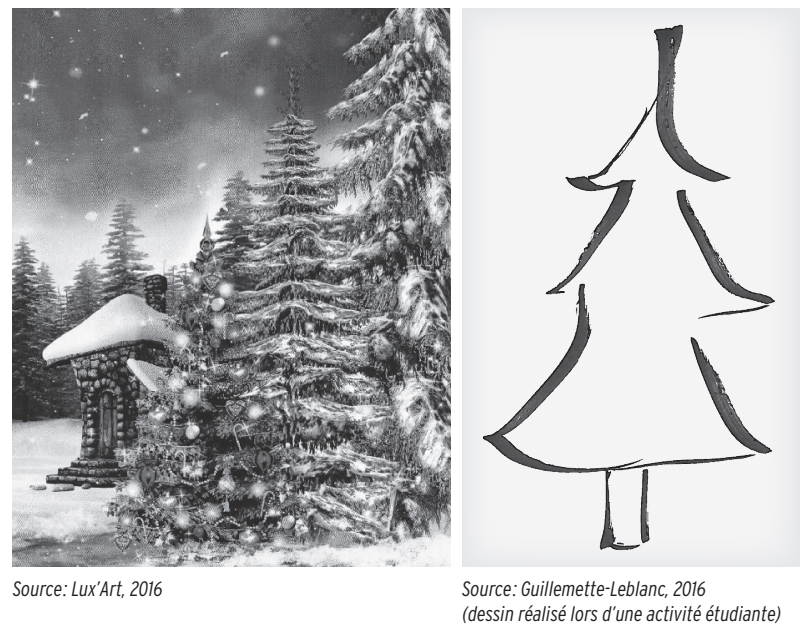

\section{Conception}

La conception est un processus relationnel autre, de pure création, qui procède elle aussi du seul travail de notre esprit, mais cette fois d'une opération cognitive sans réel lien avec quelque phénomène alors qu'elle s'emploie à aborder, signifier ou interpréter un phénomène de manière novatrice, sinon pour anticiper et planifier une relation nouvelle à son égard, c'est-à-dire pour l'inventer, sinon pour le (ré)investir.

Plus précisément, la conception est un mode d'entendement plus abstrait que les deux précédents car, dépourvue de véritable relation au phénomène ciblé, elle procède «d'une autre espèce d'idées, que les objets extérieurs [ou leur substitution] ne peuvent pas fournir» (Guibet-Lafaye, 2013: 132), et se veut projection ou «pure opération de l'esprit» (Locke, 1690/1959; Hume, 1748/2008) en vertu de laquelle l'être humain peut forger et envisager des rapports inédits ou présumés tels avec ledit phénomène. À la différence de la perception et de la représentation, qui se rapportent immédiatement à des phénomènes de l'expérience ou en émanent, la conception participe en effet d'un processus logiquement antécédent qui, de manière spéculative, s'effectue dans l'ignorance ou plutôt dans la mise à distance fortuite ou stratégique des autres déterminations de la pensée, soit de ce qu'on sait ou croit déjà savoir de ce phénomène et de nos relations avec lui. En effet, au contraire de la distinction et de la dénotation affirmatives de la perception, puis de l'évocation et de la connotation heuristiques de la représentation, la conception consiste en une opération référentielle d'une autre nature qui, au moyen d'une imagination radicale (Wunenburger, 1991), imagine à proprement parler le Réel autrement qu'il n'est en le créant, et donc nos relations avec lui, cela, à partir d'attentes ou d'une lecture autres (Kohlers, 1983). 
Ce vacuum ou cette distanciation n'indiquent pas que la conception s'effectue ex nihilo tel qu'avancé auparavant, mais bien que, réorganisant ou suppléant à divers signifiés ou signifiants préexistants, elle constitue une proposition autre structurée de significations et d’interprétations dissociées du JE réactif, et donc des sensibles, représentatifs ou sensitifs. Si, comme la représentation, la conception est modulée par un registre de sens et de valeurs, elle s'en distingue dans la mesure où ce registre s'inscrit dans un autre continuum intentionnel et dans un autre horizon opérationnel. Ainsi, lorsqu'on interroge un individu, une communauté, une association, un promoteur ou une institution gouvernementale sur sa conception de tel bassin versant, forêt, quartier historique, place publique, etc., on sonde d'abord et avant tout ses entendements et usages théoriques et idéaux de ce type de phénomène, c'est-à-dire des entendements et usages plus généraux que particuliers en ceci qu'ils en appellent essentiellement à sa vision globale de ceux-ci, et donc du bien commun ou du bien-être qui leur sont conditionnels et qui interpellent tout spécialement nos capacités d'aménagement et de planification. Stricte construction, la conception est donc, au final, proactive et même prescriptive, car foncièrement idéologique compte tenu de la vision du monde ordonnée et raisonnée que promulguent les intentions de son attitude propositionnelle (Lalande, 1926/2002).

Par exemple, je conçois ou peux concevoir un sapin baumier sans le percevoir ou le représenter, ou plutôt sans référer à ces mêmes perception et représentation, telle une création à laquelle j'attribue in abstracto divers traits, notamment formels et fonctionnels, qui peuvent ensuite être identifiés ou interprétés. Évidemment, imaginer ainsi sans référentiels ou signifiés nous apparaît à première vue impossible (nous y reviendrons avec l'affection et sa dimension non représentationnelle). Il reste que, toujours, nous inventons ou réinvestissons par conception un phénomène ou nos relations à lui à partir de rien, enfin, plus précisément de rien qui ne lui est ou ne lui serait initialement spécifique, et donc à partir de variations par déductions ou extrapolations sur des thèmes singuliers apparentés, telle une cadenza non pas unique mais unificatrice. La conception en appelle de fait à une singularité globalisante grâce à laquelle peuvent ensuite se réaliser, au diapason, les opérations de décodage et de dénotation de la perception, puis d'encodage et de connotation de la représentation, cela, une fois que pareille conception est devenue LE référentiel. Nous en voulons pour preuve l'exploitation commerciale internationale du sapin baumier québécois en lien avec la célébration de Noël pour laquelle ce seul arbre et cette seule provenance seraient sources d'authenticité.

En atteste encore la «saine» gestion de la forêt promue par la Loi sur l'aménagement durable du territoire forestier du Québec, en vigueur depuis 2010, conformément aux attentes du gouvernement québécois.

Selon cette loi, l'aménagement durable des forêts contribue plus particulièrement

1. à la conservation de la diversité biologique;

2. au maintien et à l'amélioration de l'état et de la productivité des écosystèmes forestiers;

3. à la conservation des sols et de l'eau;

4. au maintien de l'apport des écosystèmes forestiers aux grands cycles écologiques;

5. au maintien des avantages socioéconomiques multiples que les forêts procurent à la société;

6. à la prise en compte, dans les choix de développement, des valeurs et des besoins exprimés par les populations concernées (MJQ, 2010: I.2). 
Le sapin baumier est certes connu, puisque déjà bien identifié et qualifié en d'autres circonstances, mais avec la nouvelle mouture du Plan Nord publié en 2015 par le gouvernement québécois (Secrétariat au Plan Nord, 2015), par exemple, il demeure une pure création imaginaire. Il est en effet l'objet d'une conception qui émane de politiques qui ne savent pas si les forêts auxquelles on l'associe existent bel et bien au-delà des cartes qui en sont produites (la limite méridionale de la zone ciblée par le Plan Nord coupe ainsi en deux son aire de reproduction - figure 3), si son exploitation est possible autrement qu'en vertu des modèles et algorithmes qui l'affirment, quelles en sont les caractéristiques naturelles (croissance, répartition, etc.) autres que celles rapportées par les inventaires du Ministère ou des forestières privées, sinon par ce que la littérature scientifique en dit, puis comment l'administrer dans son contexte géographique (éloignement, topographie, climat, etc.) et sociopolitique (questions autochtones, main-d'œuvre, etc.) variable. Il en est ainsi car cette conception découle d'un double idéal de préservation écoculturelle (souhaitée) et de rentabilisation financière (poursuivie) des ressources forestières québécoises (qu'il soit notamment question de biomasse, de construction verte ou de bioraffinage) et, ceci entraînant cela, de l'ignorance, de la méconnaissance ou de l'interprétation intéressée, voire de la réification de typicités culturelles, économiques, environnementales, historiques, politiques et sociales du territoire québécois et de sa population.

Somme toute, ce troisième processus par lequel nous sommes en relation avec la Nature et le Territoire, et les signifions, relève d'une appréhension plus distante et éthérée que la perception, puis moins incarnée et figurative que la représentation, en ceci qu'elle est d'abord et avant tout façonnée par un advenir permettant de nous affirmer différemment, sinon de davantage nous réaliser.

\section{Affection}

Pour partie processus d'une relation primaire subséquente qui procède (i) par les sensations que génèrent notre appréhension d'un phénomène, l'affection est aussi (ii) un processus intuitif d'évocation et d'interprétation, en vertu d'un décodage-encodage afférent à une signification basique du sensible opérationnalisée par un filtre cognitif poïétique métaphorisant qui permet d'exprimer par nos émotions l'indicible de notre relation audit phénomène, ceest-à-dire pour la considérer dans toute sa profondeur de champ.

De manière plus détaillée, l'affection se distingue de la perception, de la représentation et de la conception en ceci qu'elle découle d'un mode d'entendement du Réel qui s'intéresse au sensible, c'est-à-dire à ce qui peut être l'objet d'une «intuition immédiate» (Pascal, 1668/2004). L’affection cherche ainsi à prêter voix à ce qui, affect, ambiance, atmosphère, etc., relève de l'indicible de notre relation première - au sens de primitif, d'initial - à un phénomène, soit l'ensemble de l'expérience lorsque nous sommes «en-sa-présence» (Bueno, 2013), fût-elle effective, et donc in situ, ou immatérielle et modulée par une attente (souvenir ou désir, voire besoin) qu'on veut satisfaire, et qui ne peut véritablement s'exprimer que par ce qu'on en éprouve à titre d'impressions et qui, de sitôt, occasionne des sentiments (Lalande, 1926/2002). Toute d'intériorité, l'affection fonctionne par conséquent de manière pré-cognitive (telle une réactivité psychoaffective de positionnement attitudinal) et alter-cognitive (un peu comme la représentation et la conception) en vertu d'un traitement cognitif non représentationnel pour aborder plus directement ce que les gens ressentent et non ce que ces mêmes personnes disent qu'elles ressentent (Bondi, 2009). 


\section{Figure 3 Répartition du sapin baumier}

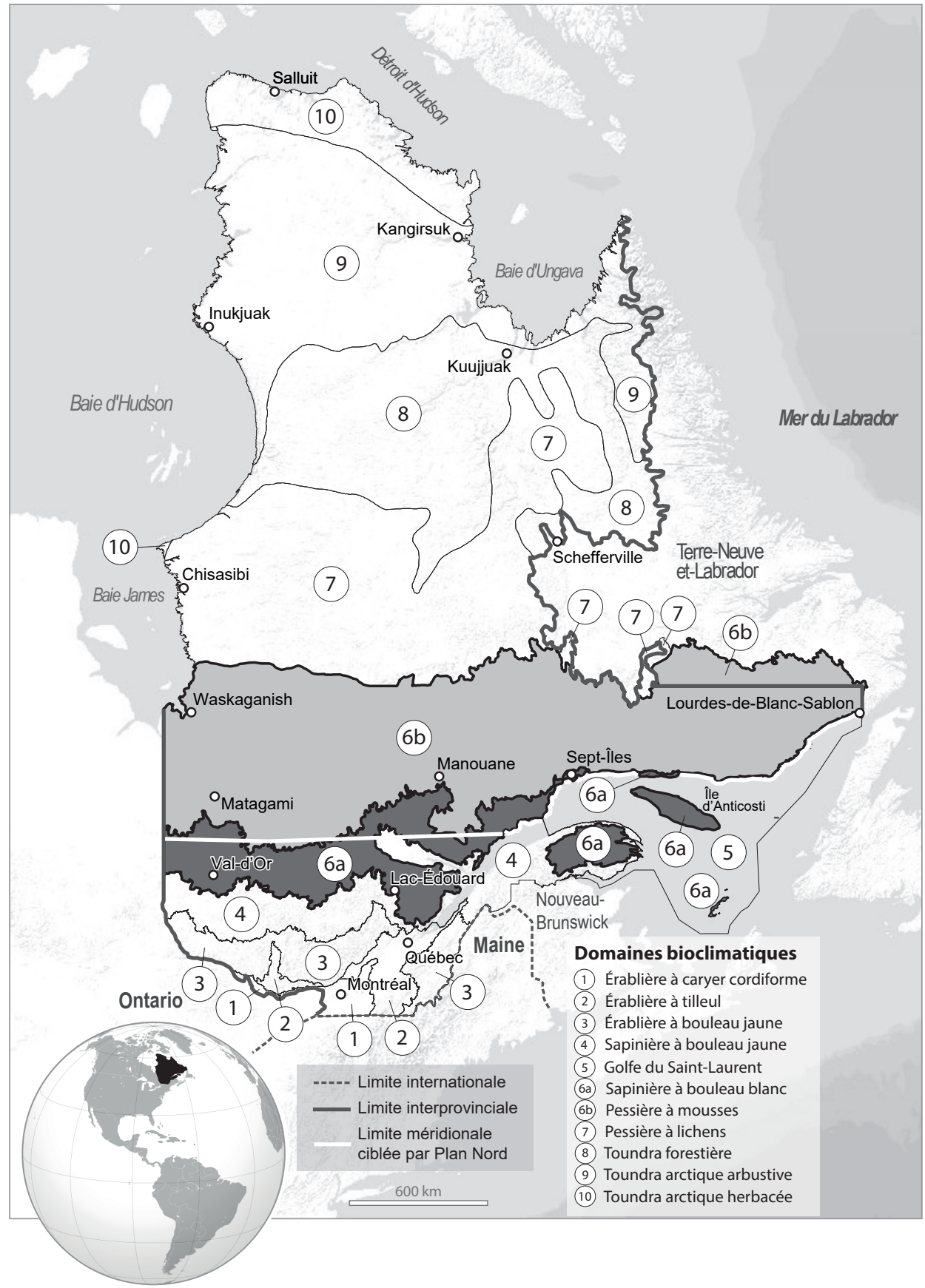

Conception: Mourad Djaballah, 2017, département de géographie de I'UQAM

Sources: ESRI, 2017; I.G.0. (données écoforestières), 2017; NOAA, 2017; USGS, 2017 
Pour ce faire, l'affection procède à partir d'une évocation et d'une interprétation propres au double registre des sensations et des émotions. Respectivement simples plaisir ou douleur, puis appréciations éthiques, morales, religieuses et sociales de ces réactions élémentaires par connotation cénesthésique (Massumi, 2002), culturelle (Stewart et Lewis, 2015) ou mémorielle (Tolia-Kelly, 2006), nos sensations et émotions s'avèrent essentielles à notre "présence en ce monde» et à sa fabrication (Merleau-Ponty, 1945/1976). Permettant en effet une contextualisation et une intelligence autres que celles opérées par les perceptions, représentations et conceptions en ceci qu'elles explorent la fibre même de l'existence humaine, c'est-à-dire ce qui rend nos existences signifiantes ou pas (Thrift, 2004 ; Davidson et Smith, 2009), nos sensations et émotions sont conditionnelles aux valeurs qu'on accorde à tel ou tel phénomène et, dès lors, aux entendements et usages qui en sont faits. Ce double pouvoir d'évocation et d'interprétation différencié de l'affection est apte à pareils approfondissement et étoffement parce qu’il repose sur un imaginaire poïétique (cf. Valéry, 1894/2016, 1919/2016; Proust, 1913/1978) qui emprunte, d'une part, à l'imaginaire reproducteur des perceptions divers éléments concrets ou dénotants bien arrêtés, notamment en termes de proximité et d'intimité, pour formuler métaphoriquement l'ineffable puis, d'autre part, à l'imaginaire (re)créateur des représentations grâce auquel on sait qu'il s'agit d'un sapin baumier et ce qu'il signifie, mais sans son pouvoir de transcendance pour approfondir, d'une manière heuristique, le surcroît de sens associé aux sensations et aux émotions. Un surcroît de sens qui, propre au bien-être auquel aspire l'affection, n'est accessible et ne peut être connoté, disions-nous, que de façon non représentationnelle, c'est-à-dire de manière moins distanciée ou idéelle que la représentation et la conception, mais encore de façon moins incarnée et immédiatement réactive que la perception.

Par exemple, quand je perçois un sapin baumier, le processus relationnel à l'œuvre me permet de prendre conscience de manière générale et nouménale que je suis face à UN sapin baumier. Le processus relationnel de l'affection me permet, lui, d'évoquer ce que je ressens à son égard, et donc de circonscrire de manière unique $\mathrm{CE}$ sapin baumier par l'entremise des sensations qu'il provoque chez moi et des émotions qui leur sont consécutives (Bueno, 2013). Cependant, il s'agit là d'une unicité qui, inaccessible à la conception car seule porteuse d'une signification plus singulière, typique à une injonction, diffère aussi de celle préconisée par la représentation en ceci que la connotation que l'affection propose est étrangère à toute opération discursive ou imaginalisante représentationnelle parce que plus intimement liée à la fonction expérientielle, et donc conjoncturelle, de NOS relations au phénomène. Moins construites, réfléchies et symboliques, donc, que celles investies par la représentation, encore que pas moins territorialisantes, si les relations qu'animent l'affection sont partiellement abstraites puisque plus développées et interprétatives que les détection et identification opérées par la perception, elles demeurent structurées par l'agrégation ou la fusion, à titre d'émotion, des messages produits par nos sens sous forme de sensations, soit une contrainte inconnue à la représentation et à la conception. Cela dit, si la sensation implique que je dois être «en-présence-de» CE sapin baumier, cela n'est pas nécessaire pour l'émotion qui, s'adonnant dans un champ affectif plus large propre à sa fonction interprétative, s'avère significative même en l'absence du sapin baumier ciblé, au même titre par ailleurs que la mémoire de la représentation mais dans une autre tonalité.

Cette consécution de l'émotion et l'importance structurante qu'on accorde dorénavant à ce type de relations expliquent par ailleurs pourquoi individus, administrateurs, élus, promoteurs économiques, associations locales, puis spécialistes (urbanistes, aménagistes, géographes, sociologues, anthropologues, thérapeutes, etc.) s'intéressent de plus en plus à l'affection. Ainsi, au sein d'une forêt les regroupant ou au contact d'un 
ou de plusieurs sapins baumiers - fût-ce le souvenir de l'expérience de ce contact -, humant leur odeur, contemplant le paysage qui se profile à travers leur canopée, écoutant le vent qui bruisse à travers leurs rameaux, nous pouvons ressentir une forme de plénitude et de sérénité inatteignables et inconnaissables sans cette immersion, mieux sans cette communion avec leur parfum, leur beauté et leur musique. Soit autant d'émotions marquantes qu'on cherche, ceci entraînant cela, (i) à reproduire, voire à pérenniser, (ii) à implanter lorsque absentes, ou (iii) à transformer sinon à éradiquer si considérées importunes ou incommodantes. Nous en voulons pour preuve les diverses vocations esthétiques (plantation et mise en valeur créant un milieu de vie agréable), éthiques (conservation ou mise en valeur soulignant la droiture environnementale des uns et des autres, qu'il s'agisse d'aménagement paysager, de projet immobilier ou de gouvernance préconisée par telle association ou administration) ou sensitives (usages thérapeutiques ou gustatifs - huiles essentielles, spas, recettes) qui peuvent ainsi être reconnues ou promulguées au sapin baumier (figure 4).

\section{Figure 4 Exemple de sensations et d'émotions que peut susciter le sapin baumier}

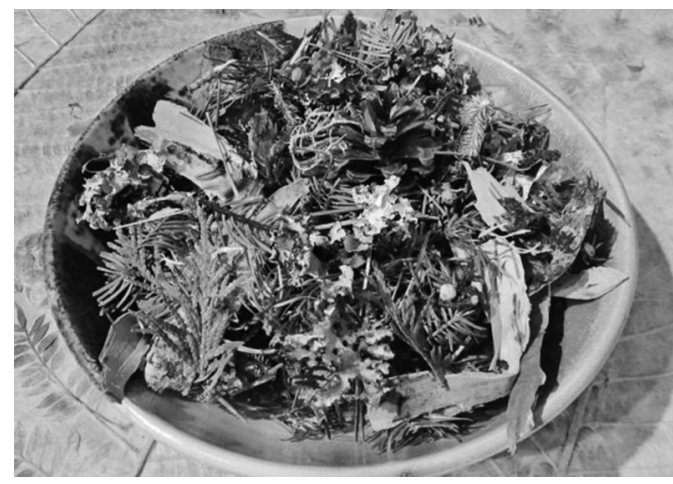

Source: Etsy, 2016

\section{Conclusion}

Dans les pages précédentes, nous avons cherché à distinguer et à articuler la perception, la représentation, la conception et l'affection

1. selon que leurs objets spécifiques s’avèrent matériels ou immatériels, dicibles ou indicibles, immédiats ou projetés, passés, présents ou futurs;

2. que leurs fonctions d'évocation procèdent par décodage ou encodage, connotation ou dénotation, voire une combinaison malléable des deux;

3. et, enfin, selon que leur appréciation du phénomène avec lequel elles sont en rapport est intérieure ou extérieure, et donc que leur positionnement vis-à-vis lui est endogène ou exogène, puis antérieur, en cours, postérieur ou conditionnel, attendu que ce positionnement peut changer en fonction du degré d'implication et de compréhension des acteurs à leur endroit, mais encore si l'imaginaire à l'œuvre est reproducteur et donc discriminant, recréateur car imaginalisant de manière heuristique et transcendante, radical puisque imaginant en créant de toute pièce, ou encore poïétique parce que prêtant voix par métaphores à l’ineffable. 
Il en a résulté quatre modes relationnels et registres de signifiance - schématisés au tableau 1 pour faciliter leur comparaison et leur usage - qui ont pu établir que nous sommes en présence d'approches chaque fois différentes des relations subjectives que nous entretenons avec la Nature, le Territoire, la Société, l'Autre. Il a toutefois aussi été constaté que leur fécondité et leur utilité sont conditionnelles aux justes entendements et usages de leurs rôles respectifs, comme de leurs éventuels maillages. ${ }^{7}$

Tableau 1 Synthèse des modalités et finalités des perception, représentation, conception et affection

\begin{tabular}{|c|c|c|c|c|}
\hline & Perception & Représentation & Conception & Affection \\
\hline Relation & primaire & secondaire & autre & primaire subséquente \\
\hline Medium & sens & esprit & esprit & sensible \\
\hline Matérialité & en présence de & en l'absence de & sans lien réel & dans l'expérience de \\
\hline Échelle & $\begin{array}{c}\text { individu } \\
\text { (générique) }\end{array}$ & $\begin{array}{c}\text { individu } \\
\text { (symbolique) }\end{array}$ & $\begin{array}{c}\text { individu } \\
\text { (société-institution) }\end{array}$ & $\begin{array}{l}\text { individu } \\
\text { (intérieur) }\end{array}$ \\
\hline $\begin{array}{l}\text { Horizon } \\
\text { temporel }\end{array}$ & présent & $\begin{array}{c}\text { passé et présent } \\
\text { sublimés }\end{array}$ & $\begin{array}{c}\text { futur ou } \\
\text { conditionnel }\end{array}$ & présent étendu \\
\hline Fonction & $\begin{array}{c}\text { dénotation affirmative } \\
\text { par décodage } \\
\text { contigu et immédiat }\end{array}$ & $\begin{array}{c}\text { connotation heuristique } \\
\text { par encodage } \\
\text { inférentiel et référentiel }\end{array}$ & $\begin{array}{l}\text { création pure } \\
\text { par projection }\end{array}$ & $\begin{array}{c}\text { appréhension } \\
\text { dénotative et } \\
\text { connotative par } \\
\text { décodage-encodage }\end{array}$ \\
\hline $\begin{array}{l}\text { Registre de } \\
\text { signification }\end{array}$ & $\begin{array}{l}\text { signification basique } \\
\text { de premier degré }\end{array}$ & $\begin{array}{l}\text { signification fine de } \\
\text { second degré, plus } \\
\text { symbolique }\end{array}$ & $\begin{array}{l}\text { signification } \\
\text { alternative, } \\
\text { liée à un idéal }\end{array}$ & $\begin{array}{c}\text { signification première, } \\
\text { plus étoffée que } \\
\text { basique }\end{array}$ \\
\hline Rôle & $\begin{array}{l}\text { traduire et structurer } \\
\text { les données sensitives }\end{array}$ & $\begin{array}{c}\text { évoquer et interpréter } \\
\text { une relation } \\
\text { antécédente par } \\
\text { notre mémoire et } \\
\text { notre imagination }\end{array}$ & \begin{tabular}{|} 
anticiper et planifier \\
de manière \\
spéculative une \\
relation nouvelle
\end{tabular} & $\begin{array}{l}\text { évoquer et interpréter } \\
\text { nos relations par nos } \\
\text { sensations et émotions }\end{array}$ \\
\hline Imaginaire & $\begin{array}{l}\text { aucun ou } \\
\text { reproducteur }\end{array}$ & (re)créateur & radical & $\begin{array}{l}\text { poïétique non } \\
\text { représentationnel }\end{array}$ \\
\hline $\begin{array}{l}\text { Objectif } \\
\text { ultime }\end{array}$ & $\begin{array}{l}\text { identifier et nommer } \\
\text { un phénomène pour } \\
\text { le (re)connaître }\end{array}$ & $\begin{array}{c}\text { qualifier le sens et } \\
\text { la valeur prêtés à un } \\
\text { phénomène pour le } \\
\text { comprendre }\end{array}$ & $\begin{array}{c}\text { aborder, signifier } \\
\text { ou interpréter de } \\
\text { manière novatrice } \\
\text { un phénomène pour } \\
\text { l'inventer, sinon pour } \\
\text { le (ré)investir afin } \\
\text { d'advenir }\end{array}$ & $\begin{array}{l}\text { exprimer l'indicible } \\
\text { d'une relation à un } \\
\text { phénomène pour la } \\
\text { considérer dans toute sa } \\
\text { profondeur de champ }\end{array}$ \\
\hline
\end{tabular}

Conception: Bédard, 2016

C'est pourquoi préconisons-nous, au final, un double principe épistémologique et méthodologique de précaution permettant

1. de déterminer d'entrée de jeu la nature du propos que commande la réflexion envisagée (et pour cela de préciser si besoin était, au-delà des appellations génériques pensée, opinion ou avis, si ce sont les perceptions, représentations, conceptions ou affections de tel ou tel phénomène qui sont recherchées) ;

7 Du nombre, signalons que les perceptions et affections sont foncièrement individuelles, plus internes et privées, davantage innées et directes, voire brutes pour construites qu'elles demeurent, alors que les représentations et conceptions le sont un peu moins, davantage externes et partagées (et donc corrélées et travaillées), liées qu’elles sont à des structures référentielles culturelles ou sociales plus larges et acquises (Kosler, 1983). 
2. puis de considérer le fait que les divers acteurs à interviewer, compte tenu de leur statut mais encore et surtout de leurs lectures plurielles et évolutives des rapports qu'ils entretiennent avec l'objet investigué, ni ne tiendront le même discours ni ne promulgueront les mêmes pratiques vis-à-vis l'espace, le territoire ou le lieu concernés, tant et si bien qu'il faut expliciter, là encore, si ce sont les perceptions, représentations, conceptions ou affections qui sont visées.

Une fois cela posé, il s'agit d'user des modes de collecte, traitement et analyse conformes aux modalités et finalités de l'un ou l'autre de ces processus afin de dégager puis d'interpréter au mieux les savoir-faire, savoir-dire et savoir-penser notre condition géographique ou territoriale que ces processus révèlent et déconstruisent.

Autrement dit, lorsque nous cherchons à savoir ce que «pense(nt) » un ou des acteur(s) vis-à-vis tel ou tel enjeu d'aménagement ou de planification territoriale, qu'il s'agisse de risques naturels, de sentiment d'appartenance, de ségrégation ou d'autonomisation sociospatiale, il nous faut être bien conscients que tous les types d'acteurs, voire tous les acteurs d'un même type, n'ont pas nécessairement les mêmes choses à dire car ils ne sont pas tous en relation de la même manière avec l'objet examiné, leurs perspectives étant dictées par l'interprétation qu'ils font de leurs rapports spécifiques audit phénomène. Il nous faut donc aborder avec soin les types d'acteurs à interviewer en étant sensibles à la nature complexe des propos qu'ils peuvent tenir, leur perception d'un objet s'avérant autre que la représentation, la conception ou l'affection qu'ils peuvent en avoir, pour intriqués que ces processus puissent être. Bien distinguer et anticiper ainsi au préalable le(s) divers type(s) de relations subjectives que les uns ou les autres peuvent avoir au lieu ou au territoire à investiguer ne peut en effet que nous aider à mieux circonscrire et comprendre (i) les acteurs à interroger et (ii) leur(s) registre(s) de signification.

\section{Remerciements}

L'auteur tient à remercier les évaluateurs anonymes du manuscrit initialement soumis, car leurs commentaires ont permis d'affiner et de bonifier le propos recherché. 


\section{Bibliographie}

ALSTON, William (2005) Perception and representation. Philosophy and Phenomenological Research, vol.70, no 2 , p. 253-289.

ANDERSON, Ben (2006) Becoming and being hopeful: Towards a theory of affect. Environment and Planning D: Society and Space, vol.24, p. 733-752.

APPLEYARD, Donald (1970) Styles and methods of structuring a city. Environment and Behavior, vol. 2, n ${ }^{\circ}$, p. 100-116.

BAILLY, Antoine S. (1974) La perception des paysages urbains - Essai méthodologique. L'Espace géographique, vol.3, nº3, p. 211-217.

BAILLY, Antoine S. (1981) La géographie de la perception dans le monde francophone: une perspective historique. Geographica Helvetica, $\mathrm{n}^{\circ} 1$, p. 14-21.

BAILLY, Antoine S. (1985) Distances et paysages: vingt ans de géographie des représentations. L'espace géographique, vol. 14, nº3, p. 197-205.

BAILLY, Antoine S. (dir.) (2005) Les concepts de la géographie humaine. Paris, Armand Colin.

BAILLY, Antoine S., FERRAS, Robert et PUMAIN, Denise (dir.) (1995) Encyclopédie de la géographie, $2^{\mathrm{e}}$ édition. Paris, Economica.

BAUD, Pascal, BOURGEAT, Serge et BRAS, Catherine (2013) Dictionnaire de géographie, $5^{\mathrm{e}}$ édition. Paris, Hatier.

BAUDRILLARD, Jean (1981) Simulacres et simulations. Paris, Galilée.

BONDI, Liz (2005) Making connections and thinking through emotions: Between geography and psychotherapy. Transactions of the Institute of British Geographers, vol. 30, nº 4, p. 433-448.

BONDI, Liz (2009) Emotional knowing. Dans Rob Kitchin et Nigel Thrift (dir.) International encyclopedia of human geography. Londres, Elsevier, p. 446-452.
BRUNET, Roger, FERRAS, Robert et THÉRY, Hervé (2005) Les mots de la géographie. Dictionnaire critique, $3^{e}$ édition revue et augmentée. Paris, La Reclus La Documentation Française.

BUENO, Otávio (2013) Perception and conception: Shaping human minds. Biosemiotics, vol. 6, n³, p. 323-336.

BUTTIMER, Anne (1974) Values in geography. Commission on College Geography Resource Paper no24. Washington D.C., Association of American Geographers.

BUTTIMER, Anne (1976) Grasping the dynamism of lifeworld. Annals of the Association of American Geographers, vol. 66, n² 2, p. 277-292.

CASTREE, Noel, KITCHIN, Rob et ROGERS, Alisdair (dir.) (2013) A dictionary of human geography. Londres, Oxford University Press.

CRESWELL, Tim (2012) Non-representational theory and me: Notes of an interested sceptic. Environment and Planning D: Society and Space, vol.30, p. 96-105.

DAVIDSON, Joyce et SMITH, Mick (2009) Emotional geographies. Dans Rob Kitchin et Nigel Thrift (dir.) International encyclopedia of human geography. Londres, Elsevier, p. 440-445.

DEBARBIEUX, Bernard (2013) Représentation II. Dans Jacques Lévy et Michel Lussault (dir.). Dictionnaire de la géographie et de l'espace des sociétés, nouvelle édition revue et augmentée. Paris, Belin, p. 866-867.

DERRIDA, Jacques (1978) La vérité en peinture. Paris, Flammarion.

DESCARTES, René (1978) Les passions de l'âme. Paris, Gallimard, [1649].

DI MÉO, Guy (2013) Perception. Dans Jacques Lévy et Michel Lussault (dir.). Dictionnaire de la géographie et de l'espace des sociétés, nouvelle édition revue et augmentée. Paris, Belin, p.759-760. 
DIXON, Deborah P. et JONES III, John Paul (1998) My dinner with Derrida: Or spatial analysis and post-structuralism do lunch. Environment and Planning A, vol. 30, p. 247-260.

DOEL, Marcus A. (1999) Poststructuralist geographies: The diabolical art of spatial science. Lanham, Rowman \& Littlefield.

DOUGLAS, Ian, HUGGET, Richard et ROBINSON, Mike (dir.) (2002). Companion encyclopedia of geography. The environment and humankind. New York, Routledge.

DRETSKE, Fred I. (1995) Naturalizing the mind. Cambridge, Massachusetts Institute of Technology Press.

DRETSKE, Fred I. (2000) Perception, knowledge, and belief. Cambridge, Cambridge University Press.

DURAND, Gilbert (1968) L'imagination symbolique. Paris, Presses Universitaires de France.

GEORGE, Pierre et VERGER, Fernand (2004) Dictionnaire de la géographie. Paris, Presses Universitaires de France.

GOULD, Peter R. (1966) On mental maps. Michigan inter-university community of mathematical geographers - Discussion Paper 9. Dans Roger M. Downs et David Stea (dir.) (1974) Image and the environment: Cognitive mapping and spatial behavior. Londres, Edward Arnold, p. 182-220.

GREGORY, Derek, JOHNSON, Ron, PRATT, Geraldine, WATTS, Michael et WHATMORE, Sarah (dir.) (2009) The dictionary of human geography, $5^{\text {th }}$ edition. Londres, Wiley-Blackwell.

GUIBET-LAFAYE, Caroline (2013) Concept. Dans Michel Blay (dir.) Dictionnaire des concepts philosophiques. Paris, Larousse et Centre national de la recherche scientifique Éditions, p. 131-133.

HUME, David (2008) Enquêtesurl'entendement humain - Enquête sur les principes de la morale. Paris, Flammarion, [1748].

JODELET, Denise (1994) Les représentations sociales. Paris, Presses Universitaires de France.
KANT, Immanuel (1998) Critique de la raison pure. Paris, Aubier, [1781].

KIRK, William (1963) Problems of geography. Geography, vol.48, n²4, p. 357-371.

KITCHIN, Rob et THRIFT, Nigel (dir.) (2009). International encyclopedia of human geography. Londres, Elsevier.

KOBAYASHI, Audrey (2009) Representation and Re-presentation. Dans Rob Kitchin et Nigel Thrift (dir.) International encyclopedia of human geography. Londres, Elsevier, p. 347-350.

KOLERS, Paul A. (1983) Perception and representation. Annual Review of Psychology, vol. 34, no 1, p. 129-166.

KOSSLYN, Stephen M. (1980) Information and mind. Cambridge, Harvard University Press.

LALANDE, André (2002) Vocabulaire technique et critique de la philosophie. Paris, Presses Universitaires de France, [1926].

LEBOEUF, Michel (2016) Arbres et plantes forestières du Québec et des Maritimes. Montréal, Éditions Michel Quintin.

LÉVY, Jacques et LUSSAULT, Michel (dir.) (2013) Dictionnaire de la géographie et de l'espace des sociétés, nouvelle édition revue et augmentée. Paris, Belin.

LEY, David (1980) Geography without man: A humanistic critique. Oxford, University of Oxford, School of Geography.

LOCKE, John (1959) An essay concerning human understanding: In two volumes. New York, Dover.

LOWENTHAL, David (1961) Geography, experience and imagination: Towards a geographical epistemology. Annals of the Association of American Geographers, vol. 51, n³ 3 p. 241-260.

LYNCH, Kevin (1960) The image of the city. Cambridge, Massachusetts Institute of Technology Press.

MARKOVÁ, Ivana (2015) Social psychology of representation. Dans James D. Wright (dir.) International encyclopedia of the social and behavioral sciences. Londres, Elsevier, p. 443-449. 
MASSUMI, Brian (2002) Parables for the virtual. Movement, affect, sensation. Durham, Duke University Press.

MAYER, Raoul Étongué et ROCHE, Yann (2012) Dictionnaire des termes géographiques contemporains. Montréal, Guérin.

MAYHEW, Susan (2009) A dictionary of geography, $4^{\text {th }}$ edition. Oxford, Oxford University Press.

McCORMAK, Derek P. (2003) The event of geographical ethics in spaces of affect. Transactions of the Institute of British Geographers, vol.28, no4, p. 488-507.

MERLEAU-PONTY, Maurice (1976) Phénoménologie de la perception. Paris, Gallimard, [1945].

MJQ (MINISTÈRE DE LA JUSTICE DU QUÉBEC) (2010) Loi sur l'aménagement durable du territoire forestier du Québec. Québec, Les publications du Québec.

MOLES, Abraham et ROHMER, Élisabeth (1972) Psychologie de l'espace. Paris, Casterman.

MORIN, Karen R. (2003) Landscape and environment: Representing and interpreting the world. Dans Sarah L. Holloway, Stephen P. Rice et Gill Valentine (dir.) Key concepts in geography. Londres, SAGE Publishing, p. 319-334.

PACHERIE, Élisabeth (2013) Perception Sensibilité, subjectif, sujet. Dans Michel Blay (dir.) Dictionnaire des concepts philosophiques. Paris, Larousse et Centre national de la recherche scientifique Éditions, p. 603-604.

PASCAL, Blaise (2004) Pensées. Paris, Gallimard, [1668].

PROUST, Marcel (1978) À la recherche du temps perdu. Paris, Gallimard, [1913].

ROUSSEAU, Jean-Jacques (1995) Euvres complètes. Paris, Gallimard, [1750-1789].

SEAMON, David (1979) A geography of the lifeworld: Movement, rest and encounter. Londres, Croom Helm.

SECRÉTARIAT AU PLAN NORD - QUÉBEC (2015) Le Plan Nord à l'horizon 2035 - Plan d'action 2015-2020 - Cadre de référence. Québec, Gouvernement du Québec.
SMITH, Alexander D. (2002) The problem of perception. Cambridge, Harvard University Press.

SPINOZA, Baruch (1993) L'Éthique, démontrée selon la méthode géométrique et divisée en cinq parties. Paris, Éditions Ivrea, [1677].

STEWART, Kathleen C. et LEWIS, Elizabeth (2015) Anthropology of affect and emotion. Dans James D. Wright (dir.) International encyclopedia of the social and behavioral sciences. Londres, Elsevier, p. 236-240.

THRIFT, Nigel (2004) Intensities of feeling: Towards a spatial politics of affect. Geografiska Annaler B, vol. 86, nº 1, p. 57-78.

THRIFT, Nigel (2008) Non-representational theory: Space, politics, affect. Londres, Routledge.

TOLIA-KELLY, Dviya P. (2006) Affect - An ethnocentric encounter? Exploring the 'universalist' imperative or emotional/ affectual geographies. Area, vol. 38, no 2 , p. 213-217.

TUAN, Yi-Fu (1971) Geography, phenomenology and the study of human nature. The Canadian Geographer, vol.15, no3, p. 181-192.

TUAN, Yi-Fu (1974) Topophilia: A study of environmental perception, attitudes, and values. Englewood Cliffs, Prentice Hall.

TUAN, Yi-Fu (1977) Space and place: The perspective of experience. Minneapolis, University of Minnesota Press.

VALÉRY, Paul (2016) Introduction à la méthode de Léonard de Vinci (1894), précédé de Notes et Digressions (1919). La Nouvelle Revue Française, vol.70, p. 5-100 [En ligne]. https://archive.org/ details/introductionla00valuoft

WACKERMAN, G. (2005) Dictionnaire de géographie. Paris, Ellipses.

WARF, Barney (2010) Encyclopedia of geography. Thousand Oaks, Sage.

WUNENBURGER, Jean-Jacques (1991) L'imagination. Paris, Presses Universitaires de France. 